\title{
Planning Step-Stress Life Tests for the Generalized Rayleigh Distribution under Progressive Type-II Censoring with Binomial Removals
}

\author{
Ali A. Ismail \\ Cairo University, Faculty of Economics \& Political Science, Department of Statistics, Giza 12613, \\ Egypt \\ aismai1100@yahoo.com (Ali A. Ismail)
}

УДК 539.4

\section{Планирование испытаний на долговечность при ступенчатой нагрузке на основе обобщенного распределения Рэлея при прогрессивном цензу- рировании типа II с биномиальными выборками}

\author{
Али А. Исмаил \\ Каирский университет, Гиза, Египет \\ Рассмотрены параметр оценки и оптимальное проектирование частично ускоренных испь- \\ таний на долговечность при ступенчатой нагрузке на основе обобщенного рэлеевского \\ распределения при прогрессивном цензурировании типа II с биномиальными выборками. В \\ качестве фактора ускорения используются максимальные оценки вероятности параметров \\ масштаба и формы, которые согласуются между собой. Построены приближенные довери- \\ тельные интерваль параметров модели и рассчитаны границь вероятности. Разработаны \\ оптимальные планы испытаний для улучшения статистического анализа. Предложены ре- \\ зультаты моделирования и числовой пример.
}

Ключевые слова: статистический анализ, оптимальный план испытаний, ступенчатая нагрузка, максимальная вероятность, обобщенное рэлеевское распределение, случайные выборки.

Introduction. Burr [1] introduced twelve families of distributions for modeling lifetime data. Among those families, Burr type X and Burr type XII have received the most attention. The Burr-type $\mathrm{X}$ distribution is also known as the generalized Rayleigh distribution (GRD). According to Burr [1], the probability density function (pdf), cumulative distribution function (cdf) and hazard function of the two-parameter GRD are defined, respectively, as below:

$$
\begin{gathered}
f(y ; \alpha, \theta)=\left(2 \theta / \alpha^{2}\right) y e^{-(y / \alpha)^{2}}\left(1-e^{-(y / \alpha)^{2}}\right)^{\theta-1}, \\
F(y ; \alpha, \theta)=\left(1-e^{-(y / \alpha)^{2}}\right)^{\theta}, \\
h(y ; \alpha, \theta)=\frac{\left(2 \theta / \alpha^{2}\right) y e^{-(y / \alpha)^{2}}\left(1-e^{-(y / \alpha)^{2}}\right)^{\theta-1}}{1-\left(1-e^{-(y / \alpha)^{2}}\right)^{\theta}}, \\
y>0, \quad \alpha>0, \quad \theta>0,
\end{gathered}
$$


where $\theta$ and $\alpha$ are the shape and scale parameters, respectively. If $\theta=1$, the GRD reduces to the traditional Rayleigh distribution. As indicated by [2-5], the GRD has been studied in many papers. Also, Surles and Padgett [6] showed that the two-parameter GRD can be used quite effectively in modeling strength data and also modeling general lifetime data.

As shown by Burr [1], if $\theta \leq 1 / 2$, the GRD has a decreasing pdf and a bathtub-type hazard function. But, when $\theta>1 / 2$, the pdf is a right-skewed unimodal function and the hazard function is an increasing function. The two-parameter GRD has several properties commonly happened in the two-parameter gamma, Weibull and generalized exponential distributions. However, when $\theta>1 / 2$, the hazard function behaves more close to the hazard function of Weibull with shape parameter greater than 1. Similar to the generalized exponential distribution and Weibull distribution, the GRD has a closed form of cdf and is very popular for dealing with censored data. Readers can refer to [4] and [7] for more detailed information about the comparison among these distributions.

In reference to the literature, there is no research work about optimum partially accelerated life test plans for the GRD under progressive type-II censoring scheme with random removals.

Because of continual improvement in manufacturing design, one often deals with products that are highly reliable with a substantially long life span. In these situations, the standard life testing methods may require time-consuming and prohibitively expensive testing time to obtain enough failure data necessary to make the desired inference. In order to assure rapid failure and then to shorten the testing period, all or some of test units may be subjected to stress conditions more severe than normal ones. Such accelerated life testing (ALT) or partially accelerated life testing (PALT) results in shorter lives than would be observed under normal operating conditions. In ALT test units are run only at accelerated conditions, while in PALT they are run at both normal (use) and accelerated conditions.

As [8] indicates, the stress can be applied in various ways, commonly used methods are step-stress and constant-stress. Under step-stress PALT (SSPALT), a test item is first run at use condition and, if it does not fail for a specified time, then it is run at accelerated condition until failure occurs or the observation is censored. But the constant-stress PALT runs each item at either use condition or accelerated condition only, i.e., each unit is run at a constant-stress level until the test is terminated. Accelerated test stresses involve higher than usual temperature, voltage, pressure, load, humidity, ..., etc., or some combination of them. The objective of a PALT is to collect more failure data in a limited time without necessarily using high stresses to all test units.

As shown from the literature, for example see [9-19], PALT has been studied under step-stress scheme by several authors. In ALT or PALT, tests are often stopped before all units fail. The estimate from the censored data is less accurate than those from complete data. However, this is more than offset by the reduced test time and expense. The most common censoring schemes are type-I and type-II censoring. Consider $n$ units placed on life test. In conventional type-I censoring, the experiment continues up to a prespecified time, $T$. Any failures that occur after that time are not observed. The termination point $T$ of the experiment is assumed to be $s$-independent of the failure times. But in conventional type-II censoring, the experimenter terminates the experiment after a prespecified number of units $m \leq n$ fail. In this scenario, only the smallest lifetimes are observed. In type-I censoring, the number of failures observed is random and the endpoint of the experiment is fixed. While the number of failures is fixed in type-II censoring and the endpoint is random.

According to [20-24], numerous articles in the literature have dealt with inference under type-I and type-II censoring for various parametric families of distributions. Conventional type-I and type-II censoring schemes do not allow removal of units at points other than the terminal point of the experiment. This paper considers a generalized censoring scheme which is progressive type-II censoring to save more time and cost 
associated with testing. It allows for the surviving units to be removed from the test at each failure time. This type of censoring will be described in the next Section.

The main purpose of this paper is to study the optimal design problem of SSPALT for units whose lifetimes follow the generalized Rayleigh distribution under progressive type-II censoring scheme with binomial removals. The rest of this paper is organized as follows: In Section 1 the test procedure and its assumptions are presented. Point and interval estimations of the model parameters are considered in Section 2. In Section 3, optimum SSPALT plans are developed under different progressive censoring schemes. In Section 4 simulation studies are conducted for illustrative purposes. Moreover, in Section 5 data analysis via a numerical example is provided. Finally, Conclusions involve some important notes and potential effort required in this track.

1. Test Procedure and Its Assumptions. As indicated by many authors, see for example [25], the type-II progressive censoring scheme has received considerable interest among the statisticians. It is a generalization of type-II censoring. Although progressively type-II censored sampling is effective in time and cost, it is not very popular in lifetime experiment. It may be due to the complicated calculation of the likelihood function.

According to [26], if an experimenter desires to remove live units at points other than the final termination point of the life test, the traditional type II censoring scheme will not be of use to the experimenter. Type II censoring does not allow for units to be lost or removed from the test at points other than the final termination point. This allowance will be desirable, as in the case of studies of wear, in which the study of the actual aging process requires units to be fully disassembled at different stages in the experiment. Intermediate removal may also be desirable when a compromise between reduced time of experimentation and the observation of at least some extreme lifetimes is sought, or when some of the surviving units in the experiment that are removed early on can be used for some other tests. As in the case of accidental breakage of experimental units or loss of contact with individuals under study, the loss of test units at points other than the termination point may also be unavoidable. These reasons lead us directly into the area of progressive censoring.

This censoring scheme can be described as follows. Suppose that $n$ units are placed on a life test and the experimenter decides beforehand the quantity $m$, the number of units to be failed. Now at the time of the first failure, $R_{1}$ of the remaining $n-1$ surviving units are randomly removed from the experiment. Continuing on, at the time of the second failure, $R_{2}$ of the remaining $n-R_{1}-2$ units are randomly withdrawn from the experiment. Finally, at the time of the $m$ th failure, all the remaining $R_{m}=n-m-R_{1}-\ldots-R_{m}-1$ surviving units are removed from the experiment. Some of the earlier work on progressive censoring was conducted by [27-29]. Recently, several articles have been published on estimating the parameters for different distribution functions, see, for example, [30-35]. A recent account on progressive censoring schemes can be found in the excellent review article introduced by [36]. In progressive type-II censoring, if $R_{1}=R_{2}=\ldots=R_{m}-1=$ $=R_{m}=0$, then $n=m$ which is the complete sampling case. But if $n-m-R_{1}-\ldots-R_{m}-$ $-1=0$, then $R_{m}=n-m$ which is the case of conventional type-II right censoring scheme.

In many reliability experiments, the pattern of removal at each failure is random. We assume that any test unit being dropped out from the life test is independent of the others but with the same removal probability (as a binomial parameter), $p$. Then, the number of test units removed at each failure time has a binomial distribution, see for example [37]. As indicated in [38], when such conditions are not satisfied or can't be assumed, one can use another distribution. In some cases, it can be assumed that the pattern of removal at each failure is fixed. But the random assumption is more realistic than the fixed one.

Now, the following assumptions are considered:

(i) $n$ identical and independent items are put on the life test;

(ii) the lifetime of each item has the GRD;

(iii) the test is finished at the $m$ th failure, where $m$ is pre-specified $(m \leq n)$; 
(iv) each of the $n$ units is first run under design condition. If it does not fail or eliminate from the test by a pre-specified time $\tau$, it is put under severe condition;

(v) at the $i$ th failure a random number of the remaining items, $R_{i}, i=1,2, \ldots, m-1$, are randomly chosen and eliminated from the test. Finally, at the $m$ th failure, the living units $R_{m}=n-m-\sum_{i=1}^{m-1} R_{i}$ are all eliminated from the test and the test is finished;

(vi) assume that an individual item being eliminated from the test is independent of the others but with the same removal probability $p$. Then, the number of items eliminated at each failure time follows a binomial distribution. That is, $R_{1} \sim \operatorname{binomial}(n-m, p)$.

For $i=2,3, \ldots, m-1, R_{i} \sim \operatorname{binomial}\left(n-m-\sum_{j=1}^{i-1} r_{j}, p\right)$ and $r_{m}=n-m-r_{1}-r_{2}-$ $-\ldots-r_{m-1}$

(vii) the lifetime, say $Y$, of an item tested under SSPALT can be expressed by

$$
Y=\left\{\begin{array}{lll}
T & \text { if } & T \leq \tau, \\
\tau+(T-\tau) / \beta & \text { if } & T>\tau,
\end{array}\right.
$$

where $T$ is the lifetime of the item under use condition, $\tau$ is the stress change time and $\beta$ is the acceleration factor, $\beta>1$. Therefore, the pdf of $Y$ can be given by

$$
f(y)= \begin{cases}0, & y \leq 0, \\ f_{1}(y) \equiv f(y ; \alpha, \theta), & 0<y \leq \tau, \\ f_{2}(y), & y>\tau,\end{cases}
$$

where

$$
f_{2}(y)=f(y ; \beta, \alpha, \theta)=\beta\left(2 \theta / \alpha^{2}\right)[\tau+\beta(y-\tau)] e^{-([\tau+\beta(y-\tau)] / \alpha)^{2}}\left(1-e^{-([\tau+\beta(y-\tau)] / \alpha)^{2}}\right)^{\theta-1},
$$

which is found by the transformation-variable procedure using $f_{1}(y)$ and the model set in (4).

Let $\left(y_{i}, r_{i}, \delta_{1 i}, \delta_{2 i}\right), i=1,2, \ldots, m$, denote the observation obtained from a progressively type-II censored sample with random removals in a SSPALT. Here $y_{(1)} \leq \ldots \leq y_{(m)}$.

Given the pre-fixed number of removals $R=\left(R_{1}=r_{1}, \ldots, R_{m-1}=r_{m-1}\right)$, the conditional likelihood function of the observations $\mathbf{y}=\left\{\left(y_{i}, r_{i}, \delta_{1 i}, \delta_{2 i}\right), i=1,2, \ldots, m\right\}$ takes the form

$$
L_{1}\left(y ; \alpha, \beta, \delta_{1 i}, \delta_{2 i} \mid \mathbf{R}=\mathbf{r}\right)=\prod_{i=1}^{m}\left\{\left[f_{1}\left(y_{i}\right)\left(S_{1}\left(y_{i}\right)\right)^{r_{i}}\right]^{\delta_{1 i}}\left[f_{2}\left(y_{i}\right)\left(S_{2}\left(y_{i}\right)\right)^{r_{i}}\right]^{\delta_{2 i}}\right\},
$$

where

$$
S_{1}(y)=1-\left(1-e^{-(y / \alpha)^{2}}\right)^{\theta}, \quad S_{2}(y)=1-\left(1-e^{-([\tau+\beta(y-\tau)] / \alpha)^{2}}\right)^{\theta} .
$$

As shown earlier, the number of items eliminated at each failure time follows a binomial distribution. Then,

$$
P\left(R_{1}=r_{1}\right)=\left(\begin{array}{c}
n-m \\
r_{1}
\end{array}\right) p^{r}(1-p)^{n-m-r_{1}} .
$$


For $i=2,3, \ldots, m-1$,

$$
P\left(R_{i}=r_{i} \mid R_{i-1}=r_{i-1}, \ldots, R_{1}=r_{1}\right)=\left(\begin{array}{c}
n-m-\sum_{j=1}^{i-1} r_{j} \\
r_{i}
\end{array}\right) p^{r_{i}}(1-p)^{n-m-\sum_{j=1}^{i} r_{j}},
$$

where $0 \leq r_{i} \leq n-m-\left(r_{1}+\ldots+r_{i-1}\right)$. Moreover, assume that $R_{i}$ is independent of $Y_{i}$ for all $i$. Then the full likelihood function can be established as

$$
L(y ; \alpha, \beta, p)=L_{1}(y ; \alpha, \beta \mid \mathbf{R}=\mathbf{r}) P(\mathbf{R}=\mathbf{r}) .
$$

Here, $\mathbf{R}=\left(R_{1}, R_{2}, \ldots, R_{m}\right)$ and $\mathbf{r}=\left(r_{1}, r_{2}, \ldots, r_{m}\right)$, and

$$
\begin{gathered}
P(\mathbf{R}=\mathbf{r})=P\left(R_{m-1}=r_{m-1}, R_{m-2}=r_{m-2}, \ldots, R_{1}=r_{1}\right)= \\
=P\left(R_{m-1}=r_{m-1} \mid R_{m-2}=r_{m-2}, \ldots, R_{1}=r_{1}\right) P\left(R_{m-2}=r_{m-2} \mid R_{m-3}=r_{m-3}, \ldots, R_{1}=r_{1}\right) \times \ldots \\
\ldots \times P\left(R_{2}=r_{2} \mid R_{1}=r_{1}\right) P\left(R_{1}=r_{1}\right) .
\end{gathered}
$$

That is,

$$
P(\mathbf{R}=\mathbf{r})=\frac{(n-m) !}{\left(n-m-\sum_{i=1}^{m-1} r_{i}\right) ! \prod_{i=1}^{m-1} r_{i} !} p^{\sum_{i=1}^{m-1} r_{i}}(1-p)^{(m-1)(n-m)-\sum_{i=1}^{m-1}(m-i) r_{i}}
$$

2. Parameter Estimation. This section deals with the process of finding the maximum likelihood estimates (MLE) of the parameters $\theta, \alpha$, and $\beta$ based on progressively type-II censored data with binomial removals. Both point and interval estimations of the parameters are considered.

2.1. Point Estimation. In this subsection, the MLEs of the model parameters are considered. From (5) we obtain the natural logarithm of the conditional likelihood function, $\ln L_{1}$, as follows:

$$
\begin{gathered}
\ln L_{1}=2 m \ln \theta-2 m \ln \alpha+m_{a} \ln \beta+\sum_{i=1}^{m_{u}} \ln y_{i}-\left(1 / \alpha^{2}\right) \sum_{i=1}^{m_{u}} y_{i}^{2}+ \\
+(\theta-1) \sum_{i=1}^{m_{u}} \ln \left[1-e^{-\left(y_{i} / \alpha\right)^{2}}\right]+\sum_{i=1}^{m_{u}} r_{i} \ln \left\{1-\left(1-e^{-\left(y_{i} / \alpha\right)^{2}}\right)^{\theta}\right\}+\sum_{i=1}^{m_{a}} \ln \psi_{i}-\left(1 / \alpha^{2}\right) \sum_{i=1}^{m_{a}} \psi_{i}^{2}+ \\
+(\theta-1) \sum_{i=1}^{m_{a}} \ln \left[1-e^{-\left(\psi_{i} / \alpha\right)^{2}}\right]+\sum_{i=1}^{m_{a}} r_{i} \ln \left\{1-\left(1-e^{-\left(\psi_{i} / \alpha\right)^{2}}\right)^{\theta}\right\},
\end{gathered}
$$

where $\psi_{i}=\tau+\beta\left(y_{i}-\tau\right)$.

Since $P(R ; p)$ does not depend on the parameters $\theta, \alpha$, and $\beta$, hence the MLEs of $\theta, \alpha$, and $\beta$ can be derived by maximizing $\ln L_{1}$ directly. Similarly, since $L_{1}(y ; \theta, \alpha, \beta \mid R=r)$ does not include the binomial parameter $p$, the MLE of $p$ can be obtained by maximizing $P(R ; p)$ directly. In particular, the MLEs of $\theta, \alpha$, and $\beta$ can be obtained by solving the following equations: 


$$
\begin{aligned}
& \frac{\partial \ln L}{\partial \theta}=\frac{2 m}{\theta}+\sum_{i=1}^{m_{u}} \ln \left[1-e^{-\left(y_{i} / \alpha\right)^{2}}\right]-\sum_{i=1}^{m_{u}} \frac{r_{i}\left[1-e^{-\left(y_{i} / \alpha\right)^{2}}\right]^{\theta} \ln \left(1-e^{-\left(y_{i} / \alpha\right)^{2}}\right)}{1-\left[1-e^{-\left(y_{i} / \alpha\right)^{2}}\right]^{\theta}}+ \\
& +\sum_{i=1}^{m_{a}} \ln \left[1-e^{-\left(\psi_{i} / \alpha\right)^{2}}\right]-\sum_{i=1}^{m_{a}} \frac{r_{i}\left[1-e^{-\left(\psi_{i} / \alpha\right)^{2}}\right]^{\theta} \ln \left(1-e^{-\left(\psi_{i} / \alpha\right)^{2}}\right)}{1-\left[1-e^{-\left(\psi_{i} / \alpha\right)^{2}}\right]^{\theta}}=0, \\
& \frac{\partial \ln L}{\partial \alpha}=-\frac{2 m}{\alpha}+\frac{2}{\alpha^{3}}\left[\sum_{i=1}^{m_{u}} y_{i}^{2}-(\theta-1) \sum_{i=1}^{m_{u}} \frac{y_{i}^{2} e^{-\left(y_{i} / \alpha\right)^{2}}}{1-e^{-\left(y_{i} / \alpha\right)^{2}}}+\right. \\
& \left.+\theta \sum_{i=1}^{m_{u}} \frac{r_{i} y_{i}^{2} e^{-\left(y_{i} / \alpha\right)^{2}}\left[1-e^{-\left(y_{i} / \alpha\right)^{2}}\right]^{\theta-1}}{1-\left[1-e^{-\left(y_{i} / \alpha\right)^{2}}\right]^{\theta}}\right]+\frac{2}{\alpha^{3}}\left[\sum_{i=1}^{m_{a}} \psi_{i}^{2}-(\theta-1) \sum_{i=1}^{m_{a}} \frac{\psi_{i}^{2} e^{-\left(\psi_{i} / \alpha\right)^{2}}}{1-e^{-\left(\psi_{i} / \alpha\right)^{2}}}+\right. \\
& \left.+\theta \sum_{i=1}^{m_{a}} \frac{r_{i} \psi_{i}^{2} e^{-\left(\psi_{i} / \alpha\right)^{2}}\left[1-e^{-\left(\psi_{\iota} / \alpha\right)^{2}}\right]^{\theta-1}}{1-\left[1-e^{-\left(\psi_{i} / \alpha\right)^{2}}\right]^{\theta}}\right]=0 \\
& \frac{\partial \ln L}{\partial \beta}=\frac{m_{a}}{\beta}+\sum_{i=1}^{m_{a}} \frac{\left(y_{i}-\tau\right)}{\psi_{i}}-\frac{2}{\alpha^{2}} \sum_{i=1}^{m_{a}}\left(y_{i}-\tau\right) \psi_{i}+ \\
& +\frac{2(\theta-1)}{\alpha} \sum_{i=1}^{m_{a}} \frac{\left(y_{i}-\tau\right) \psi_{i} e^{-\left(\psi_{i} / \alpha\right)^{2}}}{1-e^{-\left(\psi_{i} / \alpha\right)^{2}}}-\frac{2 \theta}{\alpha} \sum_{i=1}^{m_{a}} \frac{r_{i}\left[1-e^{-\left(\psi_{i} / \alpha\right)^{2}}\right]^{\theta-1}\left(y_{i}-\tau\right) \psi_{i} e^{-\left(\psi_{i} / \alpha\right)^{2}}}{1-\left[1-e^{-\left(\psi_{i} / \alpha\right)^{2}}\right]^{\theta}}=0 .
\end{aligned}
$$

Now, we have a nonlinear system of equations. It is very difficult to obtain a closed form solution. The Newton-Raphson algorithm is applied to obtain the MLEs of the unknown parameters numerically.

Independently, the MLE of the binomial parameter $p$ can be found by solving the following equation:

$$
\frac{\partial \ln L}{\partial p}=\frac{\sum_{i=1}^{m-1} r_{i}}{p}-\frac{(m-1)(n-m)-\sum_{i=1}^{m-1}(m-i) r_{i}}{1-p}=0 .
$$

Therefore, we find immediately

$$
p=\frac{\sum_{i=1}^{m-1} r_{i}}{(m-1)(n-m)-\sum_{i=1}^{m-1}(m-i-1) r_{i}} .
$$

2.2. Interval Estimation. Based on the asymptotic distributions of the MLEs of the elements of the vector of unknown parameters $\Omega=(\theta, \alpha, \beta)$, the approximate confidence intervals of the parameters can be derived. It is known that the asymptotic distribution of the MLEs of $\Omega$ is given by [39],

$$
((\hat{\theta}-\theta),(\hat{\alpha}-\alpha),(\hat{\beta}-\beta)) \rightarrow N\left(0, \mathbf{I}^{-1}(\theta, \alpha, \beta)\right),
$$


where $\mathbf{I}^{-1}(\theta, \alpha, \beta)$ is the variance-covariance matrix of the unknown parameters $\Omega=$ $=(\theta, \alpha, \beta)$. The elements of the $3 \times 3$ matrix $\mathbf{I}^{-1}, I_{i j}(\theta, \alpha, \beta), i, j=1,2,3$; can be approximated by $I_{i j}(\hat{\theta}, \hat{\alpha}, \hat{\beta})$, where

$$
I_{i j}(\hat{\Omega})=-\left.\frac{\partial^{2} \ln L(\Omega)}{\partial \Omega_{i} \partial \Omega_{j}}\right|_{\Omega=\hat{\Omega}} .
$$

Now, we get the following:

$$
\begin{aligned}
\frac{\partial^{2} \ln L}{\partial \theta^{2}}= & -\frac{2 m}{\theta^{2}}-\sum_{i=1}^{m_{u}} \frac{r_{i}\left[\psi_{u i}^{\theta}\left(\ln \psi_{u i}\right)^{2}\left(1-\psi_{u i}^{\theta}\right)+\left(\psi_{u i}^{\theta} \ln \psi_{u i}\right)^{2}\right]}{\left(1-\psi_{u i}^{\theta}\right)^{2}}- \\
& -\sum_{i=1}^{m_{a}} \frac{r_{i}\left[\psi_{a i}^{\theta}\left(\ln \psi_{a i}\right)^{2}\left(1-\psi_{a i}^{\theta}\right)+\left(\psi_{a i}^{\theta} \ln \psi_{a i}\right)^{2}\right]}{\left(1-\psi_{a i}^{\theta}\right)^{2}}
\end{aligned}
$$

where $\psi_{u i}=1-e^{-\alpha y_{i}}$ and $\psi_{a i}=1-e^{-\alpha \psi_{i}}$,

$$
\left.\begin{array}{c}
\frac{\partial^{2} \ln L}{\partial \alpha^{2}}=\frac{2 m}{\alpha^{2}}+\frac{2}{\alpha^{6}}\left\{-(\theta-1) \sum_{i=1}^{m_{u}} \frac{2 y_{i}^{4} e^{-\left(y_{i} / \alpha\right)^{2}} \psi_{u i}^{2}+2 y_{i}^{2} e^{-2\left(y_{i} / \alpha\right)^{2}}}{\psi_{u i}^{2}}+\right. \\
+\theta \sum_{i=1}^{m_{u}} \frac{r_{i} y_{i}^{2}}{\left(1-\psi_{u i}^{\theta}\right)^{2}}\left[\left(1-\psi_{u i}^{\theta}\right)\left[-2 y_{i}^{2} e^{-\left(y_{i} / \alpha\right)^{2}} \psi_{u i}^{\theta-1}-(\theta-1) 2 y_{i}^{2} e^{-2\left(y_{i} / \alpha\right)^{2}} \psi_{u i}^{\theta-2}\right]+\right. \\
\left.+2 \theta y_{i}^{2} e^{-2\left(y_{i} / \alpha\right)^{2}} \psi_{u i}^{2(\theta-1)}\right]- \\
\left.-3 \alpha^{2}\left[\sum_{i=1}^{m_{u}} y_{i}^{2}-(\theta-1) \sum_{i=1}^{m_{u}} \frac{y_{i}^{2} e^{-\left(y_{i} / \alpha\right)^{2}}}{\psi_{u i}}+\theta \sum_{i=1}^{m_{u}} \frac{r_{i} y_{i}^{2} e^{-\left(y_{i} / \alpha\right)^{2}} \psi_{u i}^{\theta-1}}{1-\psi_{u i}^{\theta}}\right]\right\}+ \\
+\frac{2}{\alpha^{6}}\left\{-(\theta-1) \sum_{i=1}^{m_{a}} \frac{2 \psi_{i}^{4} e^{-\left(\psi_{i} / \alpha\right)^{2}} \psi_{a i}^{2}+2 \psi_{i}^{2} e^{-2\left(\psi_{i} / \alpha\right)^{2}}}{\psi_{a i}^{2}}+\right. \\
+\theta \sum_{i=1}^{m_{a}} \frac{r_{i} \psi_{i}^{2}}{\left(1-\psi_{a i}^{\theta}\right)^{2}}\left[\left(1-\psi_{a i}^{\theta}\right)\left[-2 \psi_{i}^{2} e^{-\left(\psi_{i} / \alpha\right)^{2}} \psi_{a i}^{\theta-1}-(\theta-1) 2 \psi_{i}^{2} e^{-2\left(\psi_{i} / \alpha\right)^{2}} \psi_{a i}^{\theta-2}\right]+\right. \\
-3 \alpha^{2}\left[\sum_{i=1}^{m_{a}} \psi_{i}^{2}-(\theta-1) \sum_{i=1}^{m_{a}} \frac{\psi_{i}^{2} e^{-\left(\psi_{i} / \alpha\right)^{2}}}{\psi_{a i}}+\theta \sum_{i=1}^{m_{a}} \frac{r_{1} \psi_{i}^{2} e^{-\left(\psi_{i} / \alpha\right)^{2}} \psi_{a i}^{\theta-1}}{1-\psi_{a i}^{\theta}}\right]
\end{array}\right],
$$




$$
\left.\begin{array}{c}
\frac{\partial^{2} \ln L}{\partial \beta^{2}}=-\frac{m_{a}}{\beta^{2}}-\sum_{i=1}^{m_{a}} \frac{\left(y_{i}-\tau\right)^{2}}{\psi_{i}^{2}}-\frac{2}{\alpha^{2}} \sum_{i=1}^{m_{a}}\left(y_{i}-\tau\right)^{2}- \\
-\frac{2(\theta-1)}{\alpha} \sum_{i=1}^{m_{a}} \frac{\left(y_{i}-\tau\right)^{2} e^{-\left(y_{i} / \alpha\right)^{2}}\left[\psi_{a i}\left(1-(2 / \alpha) \psi_{i}^{2}\right)-(2 / \alpha) \psi_{i}^{2}\right]}{\psi_{a i}^{2}}- \\
-\frac{2 \theta}{\alpha} \sum_{i=1}^{m_{a}} \frac{r_{i}\left(y_{i}-\tau\right)^{2}}{\left(1-\psi_{a i}^{\theta}\right)^{2}}\left\{\left[\psi_{a i}^{\theta-1} e^{-\left(\psi_{i} / \alpha\right)^{2}}\left[1-(2 / \alpha) \psi_{i}^{2}\right]-\right.\right. \\
\left.\left.+(2(\theta-1) / \alpha)\left(y_{i}-\tau\right) \psi_{i}^{3} e^{-2\left(\psi_{i} / \alpha\right)^{2}} \psi_{a i}^{\theta-2}\right\}+(2 \theta / \alpha)\left(y_{i}-\tau\right) \psi_{i}^{3} e^{-2\left(\psi_{i} / \alpha\right)^{2}} \psi_{a i}^{2(\theta-1)}\right]
\end{array}\right\},
$$

and

$$
\begin{gathered}
\frac{\partial^{2} \ln L}{\partial \theta \partial \beta}=\frac{2}{\alpha} \sum_{i=1}^{m_{a}} \frac{\left(y_{i}-\tau\right) \psi_{i} e^{-\left(\psi_{i} / \alpha\right)^{2}}}{\psi_{a i}}-\frac{2}{\alpha}\left\{\sum_{i=1}^{m_{a}} \frac{r_{i}\left(y_{i}-\tau\right) \psi_{i} e^{-\left(\psi_{i} / \alpha\right)^{2}} \psi_{a i}^{\theta-1}}{1-\psi_{a i}^{\theta}}+\right. \\
\left.+\theta \sum_{i=1}^{m_{a}} \frac{r_{i}\left(y_{i}-\tau\right) \psi_{i} e^{-\left(\psi_{i} / \alpha\right)^{2}} \psi_{a i}^{\theta-1} \ln \psi_{a i}}{\left(1-\psi_{a i}^{\theta}\right)^{2}}\right\} .
\end{gathered}
$$


Thus, the approximate $100(1-\gamma) \%$ two-sided confidence bounds for $\theta, \alpha$, and $\beta$ are, respectively, given by

$$
\hat{\theta} \pm Z_{\gamma / 2} \sqrt{I_{11}^{-1}(\hat{\theta}, \hat{\alpha}, \hat{\beta})}, \quad \hat{\alpha} \pm Z_{\gamma / 2} \sqrt{I_{22}^{-1}(\hat{\theta}, \hat{\alpha}, \hat{\beta})}, \quad \hat{\beta} \pm Z_{\gamma / 2} \sqrt{I_{33}^{-1}(\hat{\theta}, \hat{\alpha}, \hat{\beta})},
$$

where $Z_{\gamma / 2}$ is the upper $(\gamma / 2)$ th percentile of a standard normal distribution.

3. Optimum Test Plans. The core intention of this section is to decide the optimal stress-change time $\tau^{*}$ based on progressive type-II censoring with binomial removals using three different progressive censoring schemes. In step-stress setting, the experimenter is often interested in estimating the mean life at use condition with high precision. The mean lifetime is an important characteristic in reliability analysis. Practically, the optimum test plans are important for improving the quality of the statistical inference. One selection optimality criterion is the D-optimality criterion. It is used to determine the optimal value of $\tau$.

The D-optimality criterion is based on the determinant of Fisher's information matrix $F$. It has been extensively used in the context of planning life test. If one is more interested in estimation with high precision, a more reasonable criterion should be D-optimality, which takes into account the overall parameter space. According to Bai et al. [40], it can be constructed in terms of the generalized asymptotic variance (GAV) of the MLEs of the model parameters. This GAV is proportional to reciprocal of the determinant of Fisherinformation matrix. So that maximizing this determinant is equivalent to minimizing GAV. The criterion function is then expressed by

$$
G A V(\hat{\alpha}, \hat{\lambda}, \hat{\beta})=\frac{1}{|\mathbf{F}|} \text {. }
$$

Hence, the optimal stress-change time $\tau^{*}$ is determined such that the GAV is minimized.

4. Simulation Studies. To demonstrate theoretical results introduced in this article, simulation studies are conducted. In order to evaluate the performance of the MLEs, the mean square error (MSE), the average confidence interval lengths of the model parameters and their coverage probabilities are obtained under three different progressive censoring schemes. Also, the influences of the sample size $n$, the observation size $m$, and the binomial parameter $p$ on the accuracy of the parameter estimates are discussed.

The simulation studies are implemented based on the following algorithm:

(i) determine the value of $n$;

(ii) determine the value of $m$,

(iii) determine the values of the parameters $\theta, \alpha, \beta$, and $p$;

(iv) determine the value of $\tau$;

(v) generate a random sample with size $n$ and observation size $m$ from the random variable $Y$ given by (4) and sort it. The generalized Rayleigh random variable can be easily generated. For example, if $U$ represents a uniform random variable from $[0,1]$, then $Y=\alpha\left[-\ln \left(1-U^{1 / \theta}\right)\right]^{1 / 2}$ has generalized Rayleigh distribution with the distribution function given by (2). The true parameters values are set to be $\theta=2, \alpha=1.5$, and $\beta=2.5$;

(vi) generate a random number $R_{1}$ from binomial $(n-m, p)$;

(vii) for $i=2,3, \ldots, m-1$, generate a random number $R_{i}$ from binomial $(n-m-$ $\left.-\sum_{l=1}^{i-1} r_{l}, p\right)$

(viii) set $R_{m}$ according to the following relation: 


$$
R_{m}=\left\{\begin{array}{lll}
n-m-\sum_{l=1}^{m-1} r_{l}, & \text { if } & n-m>\sum_{l=1}^{m-1} r_{l}, \\
0, & \text { otherwise; }
\end{array}\right.
$$

(ix) use the progressively type-II censored sample created in steps 5-8 to calculate the MLEs of the parameters $\theta, \alpha$, and $\beta$. The Newton-Raphson method is used to simultaneously solve the nonlinear equations to get the MLEs of the model parameters;

(x) steps 5-9 are replicated 10,000 times.

(xi) calculate the MSE related to the MLEs of the parameters;

(xii) construct the confidence intervals width (CIW) for each parameter with confidence level $1-\gamma=0.95$;

(xiii) steps 1-12 are implemented with different values of $n, m$, and $p$.

Tables 1-3 give the MSE of the MLEs of $\theta, \alpha$, and $\beta$. Also, these tables includes both the CIW of the model parameters and their coverage probabilities against the values of $n$ when $n=30,50,75$, and 100 and various $m$ when $p=0.10,0.25$, and 0.50 , respectively.

From the results presented in Tables $1-3$, it can be concluded that:

1. For fixed $m / n$ and $p$, the MSE associated with the parameter estimates decreases as $n$ increases.

2. For fixed $n$ and $p$, as $m$ decreases the MSE increases.

3. The effect of $m$ on the precision of the MLEs of the parameters is influenced by the value of removal probability $p$. As $p$ increases, for fixed $n$ and $m / n$, the MSE of the parameter estimates increases.

4. For fixed $m / n$ and $p$, the CIW decreases as $n$ increases. But for fixed $n$ and $m / n$, the CIW increases as $p$ increases.

5. For fixed $m / n$ and small $p$, the CP is very close to the nominal level as $n$ increases. But for fixed $n$ and $m / n$, the CP is not good as $p$ is large.

6. For fixed $n$ and $p$, as $m$ decreases, the CP is considerably lower than the nominal level.

Therefore, it can be said that as the sample size $n$ increases and the effective sample proportion $m / n$ increases, the act of the MLEs in terms of MSE become better unless $p$ is large. A higher value of $p$ leads to higher values of MSE. When $p$ increases, the experiment is terminated more quickly. But it is important to note that with a highly larger $p$ the experiments will be less informative and lead to larger standard errors in estimates. These results coincide with the note of $\mathrm{Wu}$ et al. [41]. Regarding the effect of $\tau$ on MSE of the parameter estimates, it can be said that a small value of $\tau$ gives a better estimate in the sense of having smaller MSE.

As coincided with the note of $\mathrm{Wu}$ and Huang [42], the design of an optimal life test already enables us to obtain estimations of high degree of precision. They said that in order to obtain a precise estimate of mean life, one needs to design an optimal life test. So, in this section, the optimal choice of $\tau$ is explored. Optimum test plans have been developed here numerically under different values of $n, m$, and $p$. The numerical results of the optimal stress change-time $\tau^{*}$ under different progressive censoring schemes, as well as the optimal GAV of the MLEs of the model parameters are given in Table 4. The optimal GAV is numerically obtained with $\tau^{*}$ in place of $\tau$.

From the results shown in Table 4, it can be detected that:

1. For fixed $m / n$ and $p$, both the optimal stress change-time $\tau^{*}$ and the optimal GAV of the MLEs of the model parameters decrease as the sample size $n$ increases. That is, good estimates of the model parameters are obtained.

2. For fixed $n$ and $p$, as $m$ decreases both $\tau^{*}$ and the optimal GAV of the MLEs of the model parameters increase. That is, inefficient estimates of the model parameters are obtained. 
Planning Step-Stress Life Tests ...

$\mathrm{T}$ a b 1 e 1

Results for $\boldsymbol{p}=\mathbf{0 . 1 0}$ : Average Values of MSE, CIW, and CP when $\theta, \alpha, \beta$, and $\tau$ Set at $1.5,2,2.5$, and 5 , Respectively

\begin{tabular}{|c|c|c|c|c|c|c|c|c|c|c|}
\hline$n$ & $m$ & $M S E_{\hat{\theta}}$ & $M S E_{\hat{\alpha}}$ & $M S E_{\hat{\beta}}$ & $C I W(\theta)$ & $C I W(\alpha)$ & $C I W(\beta)$ & $C P(\theta)$ & $C P(\alpha)$ & $C P(\beta)$ \\
\hline \multirow[t]{2}{*}{30} & 30 & 0.001217 & 002219 & 1.893067 & 569 & 1.475701 & 2.425164 & 0.9452 & 0.9445 & 0.9431 \\
\hline & 25 & 0.001462 & .002946 & 2.642556 & 1.296151 & 1.960679 & 2.657831 & 0.9438 & 0.9428 & 0.9422 \\
\hline \multirow[t]{6}{*}{50} & 50 & 000485 & 0.001656 & 1.013526 & 048 & 41 & 735 & 0.9463 & 0.9457 & 0.9443 \\
\hline & 45 & 0.000536 & 0.001914 & 1.131995 & 0.56 & 1.273663 & 1.363604 & 0.9445 & 0.9438 & 0.9427 \\
\hline & 40 & 0.000618 & 0.002246 & 1.330531 & 0.647662 & 1.495169 & 1.602377 & 0.9381 & 0.9374 & 0.9364 \\
\hline & 35 & 0.000796 & 0.002439 & 1.906093 & 0.833581 & 1.623662 & 1.702679 & 0.9353 & 0.9347 & 0.9336 \\
\hline & 30 & 0.001126 & .005353 & 2.512575 & 1.17 & 3.563141 & 014 & 0.9332 & 0.9326 & 0.9315 \\
\hline & 25 & 0.001703 & 0.005636 & 3.720213 & 1.78 & 3.75 & 2.12 & .9293 & 0.9286 & 0.9272 \\
\hline \multirow[t]{11}{*}{75} & 75 & 347 & 41 & 891 & 71 & 266 & 0.0 & 58 & 0.9551 & 0.9541 \\
\hline & 70 & 0.000381 & 0.001879 & 0.826313 & 0.35 & 1.04 & 683 & 0.9539 & 0.9531 & 0.9522 \\
\hline & 65 & 0.000425 & 0.002037 & 0.944028 & 0.44 & 1.107462 & 1.248992 & 0.9474 & 0.9465 & 0.9457 \\
\hline & 60 & 0.000594 & 0.002251 & 1.177313 & 0.622 & 1.152765 & 1.34 & .9446 & 0.9437 & 0.9429 \\
\hline & 55 & 0.0007 & 0.003069 & 1.48 & 0.7 & 1.3 & 1.5 & 425 & 0.9419 & 0.9408 \\
\hline & 50 & 0.000852 & 0.003 & 1.727252 & 0.89 & 1.4 & 1.731061 & 0.9383 & 0.9374 & 0.9368 \\
\hline & 45 & 0.001207 & 0.004066 & 2.687445 & 1.264673 & 571 & 1.94 & 0.9372 & 0.9363 & 0.9355 \\
\hline & 40 & 0.001512 & 0.004731 & 3.981686 & 1.584076 & 2.706533 & 412 & 0.9367 & 0.9356 & 0.9349 \\
\hline & 35 & 0.002256 & 0.005156 & 4.898563 & 2.36 & 3.148681 & 2.264005 & 0.9313 & 0.9305 & 0.9296 \\
\hline & 30 & 0 & 56 & 6.2 & 3.1 & 3.4 & 966 & 18 & 07 & 8 \\
\hline & 25 & 0.003561 & 0.005944 & 7.913035 & 3.730682 & 3.764314 & 408 & 0.9267 & 259 & 0.9251 \\
\hline \multirow[t]{16}{*}{100} & 100 & 0.000159 & 0.00667 & 703 & 682 & 0.44 & 627 & 511 & 504 & 0.9496 \\
\hline & 95 & 0.000168 & 0.000874 & 0.377281 & 0.166852 & 0.581887 & 0.791056 & 0.9524 & 0.9517 & 0.9506 \\
\hline & 90 & 0.000185 & 0.001126 & 0.398866 & 0.17 & 0.74 & 575 & 0.9481 & 0.9472 & 0.9463 \\
\hline & 85 & 0.000246 & .001281 & 0.497412 & 0.19 & 0.8 & 924 & 0.9467 & 0.9462 & 0.9447 \\
\hline & 80 & 0.000366 & 0.001405 & 0.731985 & 0.258110 & 0.935346 & 1.301911 & 0.9441 & 0.9435 & 0.9424 \\
\hline & 75 & 0.000511 & 0.001879 & 1.022658 & 0.383422 & 1.250733 & 1.552325 & 0.9426 & 0.9421 & 0.9409 \\
\hline & 70 & 0.000752 & 0.002016 & 1.395732 & 0.535291 & 1.342018 & 1.672194 & 0.9423 & 0.9411 & 0.9403 \\
\hline & 65 & 0.000931 & 0.002165 & 2.017223 & 0.787952 & 1.440661 & 1.869879 & 0.9384 & 0.9376 & 0.9366 \\
\hline & 60 & 0.001186 & 0.002412 & 2.574228 & 0.975916 & 1.605491 & 1.906743 & 0.9379 & 0.9374 & 0.9363 \\
\hline & 55 & 0.001443 & 0.003345 & 3.029566 & 1.242199 & 2.226314 & 1.956241 & 0.9354 & 0.9347 & 0.9338 \\
\hline & 50 & 0.001719 & 0.004525 & 3.550372 & 1.509162 & 3.011972 & 2.169941 & 0.9284 & 0.9276 & 0.9268 \\
\hline & 45 & 0.002103 & 0.004791 & 4.369404 & 1.801324 & 3.188484 & 2.310687 & 0.9277 & 0.9269 & 0.9262 \\
\hline & 40 & 0.002419 & 0.005329 & 5.348727 & 2.203132 & 3.547133 & 2.455186 & 0.9261 & 0.9253 & 0.9244 \\
\hline & 35 & 0.002761 & 0.005468 & 6.010147 & 2.534113 & 3.639716 & 2.847099 & 0.9257 & 0.9246 & 0.9239 \\
\hline & 30 & 0.003592 & 0.006128 & 7.634965 & 2.892334 & 4.078836 & 3.179888 & 0.9251 & 0.9244 & 0.9234 \\
\hline & 25 & 0.004183 & 0.006242 & 8.796061 & 3.763372 & 4.154546 & 3.517201 & 0.9246 & 0.9238 & 0.9231 \\
\hline
\end{tabular}

3. For fixed $n$ and $m / n$, as $p$ increases both the optimal stress change-time $\tau^{*}$ and the optimal GAV of the MLEs of the model parameters increase.

5. Data Analysis: An Illustrative Example. To show the applicability of the methodology presented in this article, a numerical example is displayed. Generalized Rayleigh model is used to fit the data set. To confirm the power of the model, we compute the Kolmogorov-Smirnov $(\mathrm{K}-\mathrm{S})$ distance between the empirical distribution function and 
T a b 1 e 2

Results for $\boldsymbol{p}=\mathbf{0 . 2 5}$ : Average Values of MSE, CIW, and CP when $\theta, \alpha, \beta$, and $\tau$ Set at $1.5,2,2.5$, and 5 , Respectively

\begin{tabular}{|c|c|c|c|c|c|c|c|c|c|c|}
\hline$n$ & $m$ & $M S E_{\hat{\theta}}$ & $M S E_{\hat{\alpha}}$ & $M S E_{\hat{\beta}}$ & $C I W(\theta)$ & $C I W(\alpha)$ & $C I W(\beta)$ & $C P(\theta)$ & $C P(\alpha)$ & $C P(\beta)$ \\
\hline \multirow[t]{2}{*}{30} & 30 & 0.001704 & 003547 & 2.082374 & 1.65 & 2682 & 2.885945 & 0.9357 & 0.9256 & 0.9148 \\
\hline & 25 & 0.002047 & 004714 & 2.906812 & 1.684996 & 2.607703 & 3.162819 & 0.9344 & 0.9239 & 0.9139 \\
\hline \multirow[t]{6}{*}{50} & 50 & 0.000679 & 0.002652 & 1.114879 & 0.660462 & 114 & 1.509795 & 0.9368 & 0.9268 & 0.9167 \\
\hline & 45 & 0.000752 & .003062 & 1.245195 & 0.729523 & 1.693972 & 1.622689 & 0.9351 & 0.9249 & 0.9144 \\
\hline & 40 & 0.000865 & .003594 & 1.463584 & 0.841961 & 1.98 & 1.906829 & 0.9287 & 0.9187 & 0.9083 \\
\hline & 35 & 0.001114 & 0.003902 & 2.096702 & 1.083655 & 2.15947 & 2.026188 & 0.9259 & 0.9163 & 0.9056 \\
\hline & 30 & 0.001576 & .008565 & 2.763833 & 1.533407 & 4.738978 & 2.244357 & .9239 & 139 & 036 \\
\hline & 25 & 0.002384 & 009018 & 4.092231 & 2.319591 & 4.98 & 2.531145 & 0.9231 & 0.9131 & 0.8994 \\
\hline \multirow[t]{11}{*}{75} & 75 & 00486 & 001826 & 0.809482 & 772 & 1.00 & 555 & .9462 & 0.9364 & 255 \\
\hline & 70 & 0.000533 & 0.003006 & 0.908944 & 0.518809 & 1.38 & 1.25 & 0.9444 & 0.9342 & 0.9236 \\
\hline & 65 & 0.000595 & 0.003259 & 1.038431 & 0.579012 & 1.472924 & 1.486371 & 0.9379 & 0.9276 & 0.9173 \\
\hline & 60 & 0.000832 & 0.003602 & 1.295044 & 0.809199 & 1.533177 & 1.601094 & 0.9352 & 0.9248 & 0.9146 \\
\hline & 55 & 0.001035 & 0.004912 & 66 & 1.00 & 25 & 1.81 & 331 & 31 & 126 \\
\hline & 50 & 0.001193 & 0.005713 & 1.899977 & 1.16 & 1.8 & 2.0 & 289 & 0.9187 & 0.9087 \\
\hline & 45 & 0.001692 & 0.006506 & 2.956193 & 1.644075 & 3.15 & 2.31 & 0.9278 & 0.9176 & 0.9074 \\
\hline & 40 & 0.002117 & 0.007572 & 4.379855 & 2.059299 & 3.599689 & 2.356694 & 0.9273 & 0.9169 & 0.9069 \\
\hline & 35 & 0.003158 & 0.008252 & 5.388419 & 3.073012 & 4.187746 & 2.694166 & 0.9221 & 0.9119 & 0.9017 \\
\hline & 30 & 39 & 54 & 952 & 09 & 56 & 182 & 0.9225 & 0.9121 & 0.9019 \\
\hline & 25 & 0.004985 & 0.009516 & 8.704339 & 4.84 & 5.00 & 076 & 0.9174 & 0.9074 & 0.8973 \\
\hline \multirow[t]{16}{*}{100} & 100 & 0.000223 & 0.001067 & 0.359373 & 4.849887 & 358 & 26 & 416 & 314 & 211 \\
\hline & 95 & 0.000235 & 0.001398 & 0.415008 & 0.216908 & 0.77 & 0.941357 & 0.9429 & 0.9327 & 0.9221 \\
\hline & 90 & 0.000259 & 0.001802 & 0.438753 & 0.228418 & 0.996589 & 1.112144 & 0.9386 & 0.9283 & 0.9179 \\
\hline & 85 & 0.000344 & 0.002050 & 0.547153 & 0.252322 & 1.134113 & 1.386261 & 0.9372 & 0.9273 & 0.9164 \\
\hline & 80 & 0.000512 & 002248 & 0.805184 & 0.335543 & 1.244016 & 1.549274 & 0.9347 & 0.9246 & 0.9141 \\
\hline & 75 & 0.000715 & 0.003006 & 1.124924 & 0.498449 & 1.663475 & 1.847267 & 0.9332 & 0.9233 & 0.9127 \\
\hline & 70 & 0.001053 & 0.003226 & 1.535305 & 0.695878 & 1.784884 & 1.989911 & 0.9329 & 0.9223 & 0.9121 \\
\hline & 65 & 0.001303 & 0.003464 & 2.218945 & 1.024338 & 1.916079 & 2.225156 & 0.9291 & 0.9188 & 0.9085 \\
\hline & 60 & 0.001662 & 0.003859 & 2.831651 & 1.268691 & 2.135303 & 2.269024 & 0.9285 & 0.9187 & 0.9082 \\
\hline & 55 & 0.002021 & 0.005352 & 3.332523 & 1.614859 & 2.960998 & 2.327927 & 0.9262 & 0.9162 & 0.9058 \\
\hline & 50 & 0.002407 & 0.007241 & 3.905409 & 1.961911 & 4.005923 & 2.582235 & 0.9191 & 0.9091 & 0.8991 \\
\hline & 45 & 0.002944 & 0.007666 & 4.806344 & 2.341721 & 4.240684 & 2.749718 & 0.9184 & 0.9084 & 0.8984 \\
\hline & 40 & 0.003387 & 0.008526 & 5.883632 & 2.864072 & 4.717687 & 2.921671 & 0.9168 & 0.9068 & 0.8967 \\
\hline & 35 & 0.003865 & 0.008749 & 6.611162 & 3.294347 & 4.840822 & 3.388048 & 0.9164 & 0.9061 & 0.8962 \\
\hline & 30 & 0.005029 & 0.009805 & 8.398462 & 3.760034 & 5.424852 & 3.784067 & 0.9158 & 0.9059 & 0.8957 \\
\hline & 25 & 0.005856 & 0.009987 & 9.675667 & 4.892384 & 5.525546 & 4.185469 & 0.9154 & 0.9053 & 0.8954 \\
\hline
\end{tabular}

the fitted distribution function when the parameters estimates are obtained by the maximum likelihood method. The result of $\mathrm{K}-\mathrm{S}$ test is $D=0.0691$ with $p=0.483$. This result observably shows that the generalized Rayleigh model provides excellent fit to the data set. Thus, it can be used successfully for modeling this data set. Assuming generalized Rayleigh distribution under progressive type-II censoring scheme with binomial removals we use $n=36, \theta=2.2, \alpha=1.25, \beta=2.5, \tau=9, m=21$, and $P=0.20$. The MSE of the MLEs of 
Planning Step-Stress Life Tests ...

T a b 1 e 3

Results for $\boldsymbol{p}=\mathbf{0 . 5 0}$ : Average Values of MSE, CIW, and CP when $\theta, \alpha, \beta$, and $\tau$ Set at $1.5,2,2.5$, and 5 , Respectively

\begin{tabular}{|c|c|c|c|c|c|c|c|c|c|c|}
\hline$n$ & $m$ & $M S E_{\hat{\theta}}$ & $M S E_{\hat{\alpha}}$ & $M S E_{\hat{\beta}}$ & $C I W(\theta)$ & $C I W(\alpha)$ & $C I W(\beta)$ & $C P(\theta)$ & $C P(\alpha)$ & $C P(\beta)$ \\
\hline \multirow[t]{2}{*}{30} & 30 & 0.002386 & 0.004966 & 2.498849 & 2.155713 & 2.355218 & 3.174545 & 0.9168 & 0.9071 & 0.8965 \\
\hline & 25 & 0.002866 & 0.006631 & 3.488174 & 2.190495 & 3.129244 & 3.479101 & 0.9157 & 0.9054 & 0.8956 \\
\hline \multirow[t]{6}{*}{50} & 50 & 0.000951 & 0.003713 & 1.337855 & 0.858601 & 1.759337 & 1.660775 & 0.9181 & 0.9083 & 0.8984 \\
\hline & 45 & 0.001053 & 0.004287 & 1.494234 & 0.94 & 2.032766 & 1.784958 & 0.9164 & 0.9064 & 0.8961 \\
\hline & 40 & 0.001211 & 0.005032 & 1.756301 & 1.094549 & 291 & 2.097512 & 0.9101 & 0.9003 & 0.8901 \\
\hline & 35 & 0.001563 & 0.005463 & 2.516042 & 1.408752 & 2.591364 & 2.228807 & 0.9074 & 0.8982 & 0.8875 \\
\hline & 30 & 0.002206 & 0.011991 & 3.316641 & 1.993429 & 5.686774 & 2.468793 & 0.9054 & 0.8956 & 0.8855 \\
\hline & 25 & 0.003338 & 0.012625 & 4.910677 & 3.015468 & 5.987131 & 2.784264 & 0.9046 & 0.8948 & 0.8814 \\
\hline \multirow[t]{11}{*}{75} & 75 & 0.000682 & 0.002556 & 0.971378 & 0.614604 & 1.211789 & 1.096211 & 0.9181 & 0.9177 & 0.9072 \\
\hline & 70 & 0.000746 & 0.004208 & 1.090733 & 0.674452 & 1.665798 & 1.380582 & 0.9164 & 0.9155 & 0.9051 \\
\hline & 65 & 0.000833 & 0.004563 & 6117 & 716 & 509 & 1.63 & 101 & 0.9091 & 0.8994 \\
\hline & 60 & 0.001165 & 0.00 & 1.554053 & 1.05 & 1.8 & 1.7 & 0.9074 & 0.9063 & 0.8963 \\
\hline & 55 & 0.001449 & 0.006877 & 1.957879 & 1.30 & 2.10 & 1.9 & 0.9054 & 0.9046 & 0.8943 \\
\hline & 50 & 0.001676 & 0.007998 & 2.279972 & 1.508887 & 2.268628 & 2.265959 & 0.9046 & 0.9003 & 0.8905 \\
\hline & 45 & 0.002369 & 0.009108 & 3.547432 & 2.137298 & 3.791411 & 2.541832 & 0.9181 & 0.8992 & 0.8893 \\
\hline & 40 & 0.002964 & 0.010601 & 5.255826 & 2.677089 & 4.319627 & 2.592363 & 0.9164 & 0.8986 & 0.8888 \\
\hline & 35 & 0.004421 & 0.011553 & 6.466103 & 3.994916 & 5.02 & 2.96 & 0.9101 & 0.8937 & 0.8837 \\
\hline & 30 & 0.005935 & 0.012676 & 8.289542 & 5.361082 & 5.476867 & 3.322241 & 0.9074 & 0.8939 & 0.8839 \\
\hline & 25 & 0.006979 & 0.013322 & 10.44521 & 6.304853 & 6.007846 & 3.876484 & 0.9054 & 0.8893 & 0.8794 \\
\hline \multirow[t]{16}{*}{100} & 100 & 0.000312 & 0.001494 & 0.431248 & 6.304853 & 0.708432 & 0.7 & 228 & 0.9128 & 027 \\
\hline & 95 & 0.000329 & 0.001957 & 0.498011 & 0.281983 & 0.928693 & 493 & 0.9241 & 0.9142 & 0.9037 \\
\hline & 90 & 0.000363 & 0.002523 & 0.526504 & 0.296943 & 1.195907 & 1.22 & 0.9198 & 0.9097 & 0.8995 \\
\hline & 85 & 0.000482 & 0.002872 & 0.656584 & 0.328019 & 1.360936 & 1.524887 & 0.9185 & 0.9088 & 0.8981 \\
\hline & 80 & 0.000717 & 0.003147 & 0.966221 & 0.436206 & 1.492819 & 1.704201 & 0.9162 & 0.9061 & 0.8958 \\
\hline & 75 & 0.001001 & 0.004208 & 1.349909 & 0.647984 & 1.996172 & 2.031994 & 0.9145 & 0.9048 & 0.8944 \\
\hline & 70 & 0.001474 & 0.004516 & 1.842366 & 0.904641 & 2.141861 & 2.188902 & 0.9142 & 0.9039 & 0.8939 \\
\hline & 65 & 0.001824 & 0.004853 & 2.662734 & 1.331639 & 2.299295 & 2.447672 & 0.9105 & 0.9004 & 0.8903 \\
\hline & 60 & 0.002327 & 0.005403 & 3.397981 & 1.649298 & 2.562364 & 2.495926 & 0.9099 & 0.9003 & 0.8931 \\
\hline & 55 & 0.002829 & 0.007493 & 3.999028 & 2.099317 & 3.553198 & 2.560723 & 0.9077 & 0.8979 & 0.8877 \\
\hline & 50 & 0.003372 & 0.010137 & 4.686491 & 2.550484 & 4.807108 & 2.840459 & 0.9007 & 0.8909 & 0.8811 \\
\hline & 45 & 0.004122 & 0.010732 & 5.767613 & 3.044237 & 5.088821 & 3.024691 & 0.9011 & 0.8902 & 0.8804 \\
\hline & 40 & 0.004742 & 0.011936 & 7.060358 & 3.723294 & 5.661224 & 3.213838 & 0.8985 & 0.8887 & 0.8788 \\
\hline & 35 & 0.005411 & 0.012249 & 7.933394 & 4.282651 & 5.808986 & 3.726853 & 0.8981 & 0.8883 & 0.8783 \\
\hline & 30 & 0.007041 & 0.013727 & 10.07815 & 4.888044 & 6.509822 & 4.162474 & 0.8975 & 0.8878 & 0.8778 \\
\hline & 25 & 0.008198 & 0.013982 & 11.61082 & 6.460099 & 6.630655 & 4.604016 & 0.8971 & 0.8872 & 0.8775 \\
\hline
\end{tabular}

$\theta, \alpha$, and $\beta$ are respectively $0.001941,0.002811$, and 1.005113 . The CIW of $\theta, \alpha$, and $\beta$ are $1.450012,2.381473$, and 2.796281 with CP $0.9360,0.9287$, and 0.9150 , respectively. Using the same settings of the life-testing experiment with different items, the results of the test design are as follows. The optimal stress change-time $\tau^{*}$ and the optimal GAV of the MLEs of the model parameters are respectively 8.3441 and 0.041532 . 
$\mathrm{T}$ a $\mathrm{b} 1$ e 4

Average Values of Optimal $\tau$ and Optimal GAV;

Considering Three Different Cases of $p, \theta, \alpha$, and $\beta$

\begin{tabular}{|c|c|c|c|c|c|c|c|}
\hline$n$ & $m$ & $\tau^{*(1)}$ & $\begin{array}{l}\text { Optimal } \\
G A V^{(1)}\end{array}$ & $\tau^{*(2)}$ & $\begin{array}{l}\text { Optimal } \\
G A V^{(2)}\end{array}$ & $\tau^{*(3)}$ & $\begin{array}{l}\text { Optimal } \\
G A V^{(3)}\end{array}$ \\
\hline \multirow[t]{2}{*}{30} & 30 & 6.1328 & 0.024364 & 8.1213 & 0.040607 & 11.1873 & 0.073092 \\
\hline & 25 & 7.3656 & 0.034012 & 11.3372 & 0.056686 & 14.8639 & 0.102035 \\
\hline \multirow[t]{6}{*}{50} & 50 & 2.4441 & 0.013044 & 4.3481 & 0.021741 & 8.3569 & 0.039133 \\
\hline & 45 & 2.7062 & 0.014569 & 4.8563 & 0.024282 & 9.6556 & 0.043707 \\
\hline & 40 & 3.1123 & 0.017125 & 5.7082 & 0.028541 & 11.3351 & 0.051374 \\
\hline & 35 & 4.0169 & 0.024531 & 8.1771 & 0.040886 & 12.3094 & 0.073594 \\
\hline & 30 & 5.6694 & 0.032338 & 10.7793 & 0.053897 & 27.0121 & 0.097014 \\
\hline & 25 & 8.5787 & 0.047882 & 15.9605 & 0.079803 & 28.4399 & 0.143645 \\
\hline \multirow[t]{11}{*}{75} & 75 & 1.7527 & 0.009472 & 3.1572 & 0.015786 & 5.7562 & 0.028415 \\
\hline & 70 & 1.9172 & 0.010635 & 3.5449 & 0.017725 & 7.9125 & 0.031904 \\
\hline & 65 & 2.1408 & 0.012152 & 4.0499 & 0.020253 & 8.3957 & 0.036449 \\
\hline & 60 & 2.9941 & 0.015152 & 5.0507 & 0.025254 & 8.7391 & 0.045456 \\
\hline & 55 & 3.7239 & 0.019089 & 6.3631 & 0.031816 & 9.9945 & 0.057268 \\
\hline & 50 & 4.3073 & 0.022231 & 7.4099 & 0.027052 & 10.7763 & 0.066689 \\
\hline & 45 & 6.0883 & 0.034587 & 11.5292 & 0.057645 & 18.0091 & 0.103761 \\
\hline & 40 & 7.6175 & 0.051243 & 17.0811 & 0.085406 & 20.5183 & 0.153732 \\
\hline & 35 & 11.3621 & 0.063047 & 21.0156 & 0.105078 & 23.8751 & 0.189141 \\
\hline & 30 & 15.2531 & 0.080824 & 26.9414 & 0.134707 & 28.0156 & 0.242473 \\
\hline & 25 & 17.9362 & 0.101842 & 33.9472 & 0.169736 & 36.5378 & 0.305525 \\
\hline \multirow[t]{16}{*}{100} & 100 & 0.8018 & 0.004205 & 1.4016 & 0.007008 & 3.3651 & 0.012614 \\
\hline & 95 & 0.8455 & 0.004856 & 1.6185 & 0.008093 & 4.4113 & 0.014567 \\
\hline & 90 & 0.9329 & 0.005233 & 1.7111 & 0.008556 & 5.6806 & 0.015443 \\
\hline & 85 & 1.2387 & 0.006402 & 2.1339 & 0.010674 & 6.4644 & 0.019205 \\
\hline & 80 & 1.8427 & 0.009421 & 3.1402 & 0.015701 & 7.0909 & 0.028262 \\
\hline & 75 & 2.5726 & 0.013162 & 4.3872 & 0.021936 & 9.4818 & 0.039485 \\
\hline & 70 & 3.7882 & 0.017963 & 5.9877 & 0.029939 & 10.1741 & 0.053889 \\
\hline & 65 & 4.6877 & 0.025962 & 8.6539 & 0.043278 & 10.9224 & 0.077885 \\
\hline & 60 & 5.9804 & 0.033132 & 11.0434 & 0.055217 & 12.1717 & 0.099391 \\
\hline & 55 & 7.2705 & 0.038993 & 12.9976 & 0.064988 & 16.8788 & 0.116978 \\
\hline & 50 & 8.6661 & 0.045694 & 15.2312 & 0.076156 & 22.8340 & 0.137081 \\
\hline & 45 & 10.5942 & 0.056236 & 18.7454 & 0.093727 & 24.1722 & 0.168709 \\
\hline & 40 & 12.1873 & 0.068838 & 22.9461 & 0.114731 & 26.8916 & 0.206515 \\
\hline & 35 & 13.9062 & 0.077355 & 25.7849 & 0.128925 & 27.5932 & 0.232064 \\
\hline & 30 & 18.0951 & 0.98264 & 32.7547 & 0.163774 & 35.9226 & 0.294792 \\
\hline & 25 & 21.0697 & 0.113206 & 37.7354 & 0.188677 & 39.4965 & 0.339619 \\
\hline
\end{tabular}

(1) When $p=0.10$ and $\theta, \alpha$, and $\beta$ set at $1.5,2$, and 2.5 , respectively;

(2) when $p=0.25$ and $\theta, \alpha$, and $\beta$ set at $1.5,2$, and 2.5 , respectively;

(3) when $p=0.50$ and $\theta, \alpha$, and $\beta$ set at $1.5,2$, and 2.5 , respectively.

Conclusions. The issue of progressive type-II censoring has received care in the past few years. The GRD can be used quite effectively in modeling strength data and also modeling general lifetime data. MLEs of parameters of the GRD were discussed using 
progressively type-II censored data with binomial removals. The performance of the MLEs was assessed numerically. In addition, the roles of sample size $n$, failure size $m$, and removal probability $p$ toward the accuracy of estimations were explored via simulation studies. It can be concluded that as both the sample size $n$ and the effective sample fraction $m / n$ increase, the performance of the MLEs become better unless $p$ is large.

Moreover, statistically optimum step-stress partially accelerated life test plans have been developed. The optimality criterion adopted is the minimization of the GAV of the MLEs of the model parameters. That is, $\tau^{*}$ is obtained such that the GAV is minimized. Thus, the optimal design of the life tests can be considered as a technique to improve the quality of the statistical inference. This issue agrees with the annotation of $\mathrm{Wu}$ and Huang [38]. In order to obtain a precise estimate of mean life, one needs to design an optimal life test. As a future work, the Bayesian inference in the case of SSPALT under the same censoring schemes proposed in this paper will be considered. Also, the optimum test plans will be explored under constant-stress PALT using progressively type-II censored data which is an extension to the work of Ismail [43].

\section{Резюме}

Розглянуто параметр оцінки і оптимальне проектування частково прискорених випробувань на довговічність при ступеневому навантаженні на основі узагальненого релеївського розподілу при прогресивному цензуруванні типу II з біноміальними виборками. Як фактор прискорення використовуються максимальні оцінки імовірності параметрів масштабу і форми, які узгоджуються між собою. Побудовано наближені довірчі інтервали параметрів моделі і розраховано границі імовірності. Розроблено оптимальні плани випробувань із метою покращання статистичного аналізу. Запропоновано результати моделювання і числовий приклад.

1. I. W. Burr, "Cumulative frequency distribution," Ann. Math. Stat., 13, No. 2, 215-232 (1942).

2. Y. L. Lio, D.-G. Chen, and T.-R. Tsai, "Parameter estimations for generalized Rayleigh distribution under progressively type-I interval censored data," Open J. Stat., 1, 46-57 (2011).

3. M. Z. Raqab and M. T. Madi, "Inference for the generalized Rayleigh distribution based on progressively censored data," J. Stat. Plan. Infer., 141, 3313-3322 (2011).

4. D. Kundu and M. Z. Raqab, "Generalized Rayleigh distribution: Different methods of estimations," Comput. Stat. Data Anal., 49, 187-200 (2005).

5. J. Li and L. Ma, "Inference for the generalized Rayleigh distribution based on progressively type-II hybrid censored data," J. Inf. Comput. Sci., 12, 1101-1112 (2015).

6. J. G. Surles and W. J. Padgett, "Inference for reliability and stress-strength for a scaled Burr type X distribution," Lifetime Data Anal., 7, 187-200 (2001).

7. M. Z. Raqab and D. Kundu, "Burr type X distribution: Revisited" (2003), http:// home.iitk.ac.in/kundu/paper118.pdf.

8. W. Nelson, Accelerated Testing: Statistical Models, Test Plans, and Data Analysis, John Wiley \& Sons, New York (1990).

9. P. K. Goel, Some Estimation Problems in the Study of Tamered Random Variables, Technical Report No. 50, Department of Statistics, Carnegie Mellon University, Pittsburgh, PA (1971).

10. M. H. Degroot and P. K. Goel, "Bayesian and optimal design in partially accelerated life testing," Nav. Res. Logist. Q., 26, No. 2, 223-235 (1979). 
11. G. K. Bhattacharyya and Z. Soejoeti, "A tampered failure rate model for step-stress accelerated life test," Commun. Stat. Theor. Meth., 18, No. 5, 1627-1643 (1989).

12. D. S. Bai and S. W. Chung, "Optimal design of partially accelerated life tests for the exponential distribution under type-I censoring," IEEE Trans. Reliab., 41, 400-406 (1992).

13. D. S. Bai, S. W. Chung, and Y. R. Chun, "Optimal design of partially accelerated life tests for the lognormal distribution under type-I censoring," Reliab. Eng. Syst. Safe., 40, 85-92 (1993).

14. A. A. Ismail, The Test Design and Parameter Estimation of Pareto Lifetime Distribution under Partially Accelerated Life Tests, Ph.D. Thesis, Department of Statistics, Faculty of Economics \& Political Science, Cairo University, Egypt (2004).

15. H. M. Aly and A. A. Ismail, "Optimum simple time-step stress plans for partially accelerated life testing with censoring," Far East J. Theor. Stat., 24, No. 2, 175-200 (2008).

16. A. A. Ismail, "Bayes estimation of Gompertz distribution parameters and acceleration factor under partially accelerated life tests with type-I censoring," J. Stat. Comput. Sim., 80, No. 11, 1253-1264 (2010).

17. A. A. Ismail, "Estimating the parameters of Weibull distribution and the acceleration factor from hybrid partially accelerated life test," Appl. Math. Model., 36, No. 7, 2920-2925 (2012).

18. A. A. Ismail, "Inference for a step-stress partially accelerated life test model with an adaptive Type-II progressively hybrid censored data from Weibull distribution," $J$. Comput. Appl. Math., 260, 533-542 (2014).

19. A. A. Ismail, "Statistical inference for a step-stress partially-accelerated life test model with an adaptive Type-I progressively hybrid censored data from Weibull distribution," Stat. Pap., 57, No. 2, 271-301 (2016).

20. A. C. Cohen, Truncated and Censored Samples: Theory and Applications, Marcel Dekker (1991).

21. Ali A. Ismail, "Estimating the generalized exponential distribution parameters and the acceleration factor under constant-stress partially accelerated life testing with type-II censoring," Strength Mater., 45, No. 6, 693-702 (2013).

22. Ali A. Ismail, "On designing constant-stress partially accelerated life tests under time-censoring," Strength Mater., 46, No. 1, 132-139 (2014).

23. Ali A. Ismail, "Bayesian estimation under constant-stress partially accelerated life test for Pareto distribution with type-I censoring," Strength Mater., 47, No. 4, 633-641 (2015).

24. Ali A. Ismail, "Optimum failure-censored step-stress life test plans for the Lomax distribution," Strength Mater., 48, No. 3, 437-443 (2016).

25. J. F. Lawless, Statistical Models and Methods for Lifetime Data, John Wiley \& Sons, New York (1982).

26. N. Balakrishnan and R. Aggarwala, Progressive Censoring: Theory, Methods, and Applications, Birkhäuser, Boston (2000).

27. A. C. Cohen, "Progressively censored samples in the life testing," Technometerics, 5, No. 3, 327-339 (1963).

28. N. R. Mann, "Best linear invariant estimation for Weibull parameters under progressive censoring," Technometrics, 13, No. 3, 521-533 (1971). 
29. D. R. Thomas and W. M. Wilson, "Linear order statistic estimation for the two-parameter Weibull and extreme value distributions from type-II progressively censored samples," Technometrics, 14, No. 3, 679-691 (1972).

30. R. Viveros and N. Balakrishnan, "Interval estimation of parameters of life from progressively censored data," Technometrics, 36, No. 1, 84-91 (1994).

31. U. Balasooriya and N. Balakrishnan, "Reliability sampling plans for log-normal distribution based on progressively censored samples," IEEE Trans. Reliab., 49, 199-203 (2000).

32. N. Balakrishnan and N. Kannan, "Point and interval estimation for the logistic distribution based on progressively type-II censored samples," in: N. Balakrishnan and C. R. Rao (Eds.), Handbook of Statistics, Vol. 20, Advances in Reliability, Elsevier (2001), pp. 431-456.

33. S. J. Wu and C. T. Chang, "Parameter estimations based on exponential progressively type-II censored data with binomial removals," Int. J. Inf. Manag. Sci., 13, No. 3, 37-46 (2002).

34. S. J. Wu and C. T. Chang, "Inference in the Pareto distribution based on progressive Type-II censoring with random removals," J. Appl. Stat., 30, No. 2, 163-172 (2003).

35. N. Balakrishnan, N. Kannan, C.-T. Lin, and H. K. T. Ng, "Point and interval estimation for Gaussian distribution, based on progressively type-II censored samples," IEEE Trans. Reliab., 52, 90-95 (2003).

36. N. Balakrishnan, "Progressive censoring methodology: An appraisal (with discussions)," Test, 16, 211-296 (2007).

37. S. K. Tse, C. Yang, and H. K. Yuen, "Statistical analysis of Weibull distributed lifetime data under Type-II progressive censoring with binomial removals," J. Appl. Stat., 27, 1033-1043 (2000).

38. Ali A. Ismail, "Inference in the generalized exponential distribution under partially accelerated tests with progressive Type-II censoring," Theor. Appl. Fract. Mech., 59, 49-56 (2012).

39. R. C. Miller, Survival Analysis, John Wiley \& Sons, New York (1981).

40. D. S. Bai, J. G. Kim, and Y. R. Chun, "Design of failure-censored accelerated life test sampling plans for lognormal and Weibull distributions," Eng. Optimiz., 21, 197-212 (1993).

41. S. J. Wu, Y. P. Lin, and Y. J. Chen, "Planning step-stress life test with progressively type-I group-censored exponential data," Stat. Neerl., 60, 46-56 (2006).

42. S. J. Wu and S. R. Huang, "Optimal progressive group-censoring plans for exponential distribution in presence of cost constraint," Stat. Pap., 51, 431-443 (2010).

43. Ali A. Ismail, "Planning failure-censored constant-stress partially accelerated life test," J. Syst. Eng. Electron., 26, 644-650 (2015). 